\title{
Using individual data to characterize emotional user experience and its memorability: focus on gender factor
}

\author{
Romain Cohendet*, Anne-Laure Gilet ${ }^{\dagger}$, Matthieu Perreira Da Silva* and Patrick Le Callet* \\ * IRCCyN \\ University of Nantes, France \\ Email: \{romain.cohendet, matthieu.perreiradasilva, patrick.le-callet\}@univ-nantes.fr \\ $\dagger$ LPPL \\ University of Nantes, France \\ Email: anne-laure.gilet@univ-nantes.fr
}

\begin{abstract}
Delivering the same digital image to several users is not necessarily providing them the same experience. In this study, we focused on how different affective experiences impact the memorability of an image. Forty-nine participants took part in an experiment in which they saw a stream of images conveying various emotions. One day later, they had to recognize the images displayed the day before and rate them according to the positivity/negativity of the emotional experience the images induced. In order to better appreciate the underlying idiosyncratic factors that affect the experience under test, prior to the test session we collected not only personal information but also results of psychological tests to characterize individuals according to their dominant personality in terms of masculinity-femininity (Bem Sex Role Inventory) and to measure their emotional state. The results show that the way an emotional experience is rated depends on personality rather than biological sex, suggesting that personality could be a mediator in the well-established differences in how males and females experience emotional material. From the collected data, we derive a model including individual factors relevant to characterize the memorability of the images, in particular through the emotional experience they induced.
\end{abstract}

\section{INTRODUCTION}

The overwhelming - and growing - number of digital images shared every day makes highly desirable the possibility to automatically predict how relevant they are for the user to whom they are intended. The memorability of an image has recently been proposed as a relevant indicator to help picking out the most meaningful images from a photo collection or a video [1]. The image memorability consists in the faculty of an image to be recalled after a period of time [2]. It appears from recent studies that it is possible to predict the degree of image's memorability quite well [1], [3], [4]. On the other hand, the emotion an image induces can also be regarded as interesting for automatically characterizing its relevance for a particular observer, within a particular context. Indeed, it has been shown that emotional information can be computationally inferred from the image to some extent (for an overview, see [5]).

While existing research on the prediction of memorability have not taken into account the emotional experience induced by the images, psychological studies investigating the links between emotion and memory suggest that it would be inter- esting. In particular, it has been shown that the valence (i.e. the hedonic tone, ranging from pleasant to unpleasant) of an emotional experience induced by an image is closely related to its memorization (e.g. [6]-[8]). As emotional information can be computationally extracted from image, it could be used to enhance automatic memorability prediction. This would, however, require to know the links between emotional information an image conveys and its memorability.

However, delivering the same image to several users is not necessarily providing them the same experience; accordingly, the memorability of an image, since it depends on the experience, changes in some extent along with individual and context. The consideration of individual factors may help to increase the accuracy of emotional information extraction and memorability prediction, by integrating idiosyncrasy in the equation. To achieve this goal, studies are necessary to better understand how the user factors impact the emotional experience induced by an image and its memorability, and to model them with the aim of integrating information related to the user in the optimization of the digital experience, so that they operate in a personalized way.

In the psychological literature, several individual factors have been highlighted for their impact on both the emotional experience induced by an image and its memorability, including age, gender, affective state (both stable or trait emotions, and current or state ones), and level of fatigue.

Some studies have shown that age is related to the way emotional valence of an experience induced by an image is rated. For example, Grühn et Scheibe (2008) found that older adults perceived negative pictures as more negative than young adults did [7]. Furthermore, the well documented memory boost for negative images sounds to be less important for older adults than for young adults (maybe because of the greater investment in emotion regulation with age) [6], [8].

The literature also suggests that there are differences in the way emotional images are processed by males and females. In particular, females seem to experience the negativity of an image more intensively than males. For example, in a neuroimaging study Wrase et al. (2003) found that females showed a stronger brain activation for affectively negative pic- 
tures in the anterior and medial cingulate gyrus [9]. Similarly, in a neurophysiological study Lithari et al. (2009) found that unpleasant stimuli evoked a greater ERP amplitudes in females relatively to males [10].

These results seem to suggest that biological sex determines the fact of being more responsive to negative emotional stimuli. However, this difference in sensitivity could be not directly related to biological sex, but to the individual's personality - which could be more or less often associated with an emotional component depending on whether the person is a male or a female - largely dependent on the gender role expected by society [11]. Several questionnaires have been developed to measure gender roles, the most famous being the Bem Sex Role Inventory (BSRI) [12], [13]. It assesses how individuals identify themselves psychologically, and in particular measures the masculinity-femininity. By using BSRI, one could test if the dominant personality (masculine or feminine) or the biological sex (male or female) have the greatest impact on the way emotional induced by an image is experienced.

Trait emotions may participate in the emotional state induced by an stimulus. An example is the mood-congruence effect, which refers to the tendency of individuals to retrieve information more easily when it has the same emotional content as their emotional state. This bias is typically found in depression, where a depressed mood increases the tendency to remember negative events (e.g. [14], [15]). In the same way, state emotion may play a role in emotion-congruent memory. The priming by a stimulus that has the same emotional content as the target reduces the processing time of the latter, a phenomenon known as the affective priming effect (e.g. [16]). In a condition where images are displayed in a stream, the emotion induced by an image could, for instance, interfere with the emotional experience induced by the next image.

Finally, it has long been known that the fatigue level decreases brain performance in general, and affects memory and affective state in particular. By way of an extreme example, sleep deprivation is known to have critical effect both on mood and memory [17].

\section{A. Purpose of the study}

In order to better appreciate the underlying idiosyncratic factors that affect the emotional experience induced by an image and its memorability, prior to the test session we collected personal information about the participants (namely, their age, gender, emotional state, fatigue level, and some personality traits) through questions and self-assessment psychological tests. Then participants performed an encoding memory task, in which they saw a stream of images. The next day, they performed a recognition test, where the images viewed the day before were displayed among new images; they had to recognize the target images, and then rate them according to the valence of the emotional experience they induced. In the analysis of the results, we compare biological sex versus gender role influences on how the emotional experience is rated. Then, we derive a model from the data to link memorability to all factors for which we obtained measures.

\section{METHOD}

Forty-nine participants (18-41 years of age; $\bar{x}=$ 23.15; $S D=5.56 ; 59 \%$ of them female) compensated for their participation took part in the experiment. They had never participated in a psychological experiment before. The experiment was conducted in two days, in IRCCyN laboratory facilities (Nantes, France), in an experimental room specifically designed and equipped for human assessment of image quality. Upon their arrival, they passed a visual acuity test (Monoyer' scales) and a color vision test (Ishihara color vision test) to ensure no vision problem interfered with the test. Then they answered questionnaires, and performed a memory encoding task in which they saw a stream of images (see Figure 1(a)). The next day, they performed a recognition task in which they had to recognize the 50 target images (i.e.viewed the day before), while they were displayed among 200 new images (see Figure 1(b)). Then, they rated targets according to the positivity/negativity of the emotional experience they induced (see Figure 1(c)).

\section{A. Stimuli and display equipment}

In order to induce in participants a wide range of emotions, we used 625 images randomly selected from the International Affective Picture System (IAPS) [18]. This database provides researchers with a set of 1196 color standardized photographs (for some modified) rated along the dimension of arousal and valence. The images were displayed on a 40 inch monitor (TV-LOGIC LVM401) with a display resolution of 1920x1080. The participants were seated at a distance of 150 centimeters from the screen (three times the screen height). The $1024 \times 768$ images were centered on a black background; at a viewing distance of $150 \mathrm{~cm}$ the stimuli subtended 18.85 degrees of vertical visual angle.

1) Questionnaires: To collect participants' personal data on the dimensions we were interested in, we used different psychological tools.

We used the BSRI [13] to measure the masculinityfemininity. This self-assessment questionnaire consists of 60 items, for which an individual must say how well they match his personality on a scale ranging from 1 (never or almost never true) to 7 (always or almost always true). The measure is based on descriptors that refer to different traits and behaviors that men and women consider valued for a woman or a man - that is to say, the socially accepted gender characteristics. It includes 20 'feminine' items (e.g. gentle, compassionate), 20 'masculine' items (e.g. self reliant, acts as a leader) and 20 filler trait thought to be gender neutral (e.g. sincere, adaptable). The BSRI outputs a categorization of the personality of the individual as 'masculine', 'feminine', 'androgynous' (i.e. both male and female poles highly developed) or 'undifferentiated' (i.e. both male and female poles underdeveloped).

The International Positive and Negative Affect Schedule Short Form (I-PANAS-SF) [19] was used to measure the longterm affective state (trait emotions) of the participant (during the past year). It is a shortened version of the PANAS [20] which contains 10 items, intended to international use with participants whose native language is not English. Participants completing the I-PANAS-SF are asked to rate the extent to which they experience the regarded item on a 7-point Likert Scale ranging from 1 (very slightly/never) to 7 (very much/often). I-PANAS-SF provides results on two dimensions: positive affect and negative affect. 
(a)

Training
phase
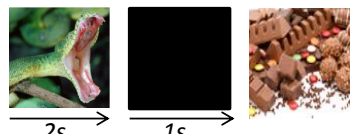

Encoding task (Day 1)

$15 \min$
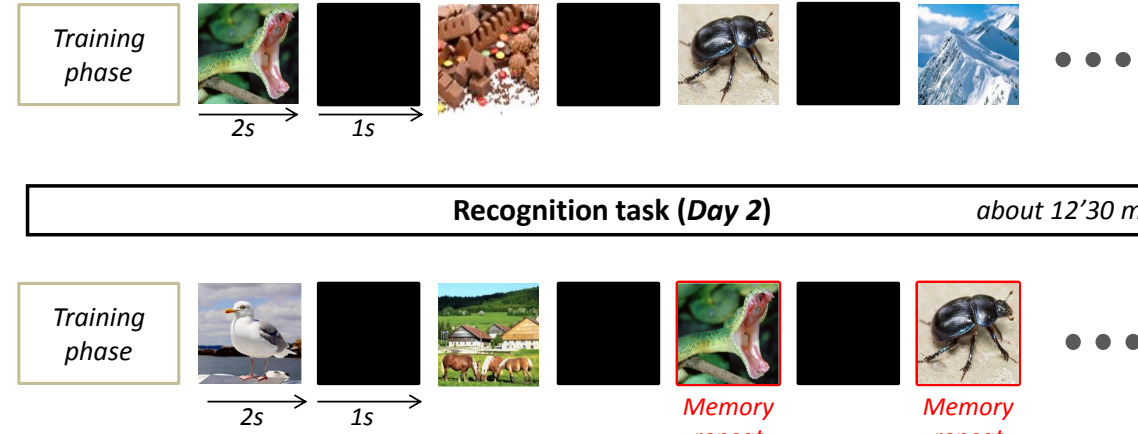

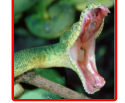

Memory

repeat
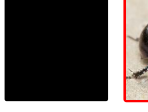

Memory

repeat

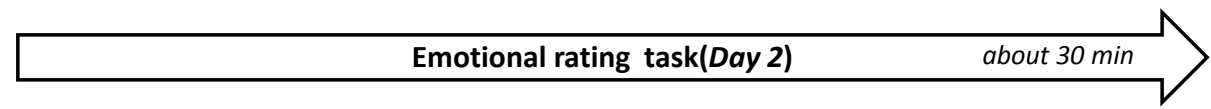

(c)
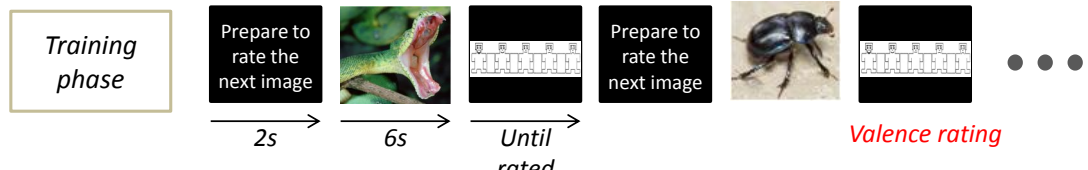

Fig. 1. The different experimental tasks. (a) The memory encoding task the first day. (b) The recognition task around 24 hours later (targets are part of the precedent day images). (c) Emotional rating task for previous target images. For the three tasks, the images were displayed in randomized order.

The mood matrix proposed by Eich and Metcalfe [21] was used to assess the participants' current mood at the beginning of each experimental phase. This matrix consists of (9x9) 81 squares; it measures simultaneously the arousal - which corresponds to the degree of physiological activation, ranging from somnolence to high awareness [22] - (vertical axis) and valence (horizontal axis) emotional dimensions. The center of the matrix would represent a neutral mood (neither positive nor negative and neither aroused nor unaroused). Participants were asked to tick the square that best reflected their current feelings (e.g. a participant who experienced a positive and highly arousing feelings should tick a square to the top right of the matrix).

For the purpose of measuring the participants' level of fatigue, we used a single item extracted from the PANAS (namely "Indicate the extent you are feeling tired." 1: "Very tired' to 5: 'Absolutely rest').

Finally, for the emotional rating task, in which the participants had to rate in terms of positivity/negativity the emotional experience induced by the target images, we used the Self Assessment Manikin (SAM) scale for valence [23]. This nonverbal pictorial assessment technique consists of an affective rating scale in which degrees are represented by nine graphic figures depicting values along the emotional dimension of valence (ranging from 1 - very negative - to 9 - very positive - , with the figure at the center corresponding to a neutral neither positive nor negative - emotion).

\section{RESULTS}

We present results in two parts: first, we test the hypothesis that dominant personality (masculine or feminine) affects more than biological sex the way individuals rate the positivitynegativity of an emotional experience; second, we derive a mathematical model from all data to link individual factors to the probability of an image to be recognized when it is repeated. We report the discussion of the results in the next part.

\section{A. BSRI scoring}

Participants' self-descriptions through BSRI items were scored using a median-split technique based on scores derived from our sample, as recommended by Bem in 1977 [12] following Spence et al.' criticism about her original procedure, which did not discriminate between individuals who scored high, and those who scored low on Feminine and Masculine scales [24]. The BSRI categorizes individuals into four possibilities: masculine, feminine, androgynous and undifferentiated. A sex-typed classification (masculine or feminine) is the result of scoring above the median in one gender and below the median in the other. Androgynous categorization occurs when a subject scores above $50 \%$ of the group in both masculine and feminine categories. Undifferentiated is the result of scoring below the median in both masculine and feminine categories (seen as the result of extremely low masculine and feminine traits).

The results of the categorization, according to the biological sex of the participants, are reported in table I. We can note that $48 \%$ of the male participants were classified into 'Masculine' category, and $24 \%$ into 'Feminine' category, while $57 \%$ of female participants were classified into 'Feminine' category and $32 \%$ into 'Masculine' category. 

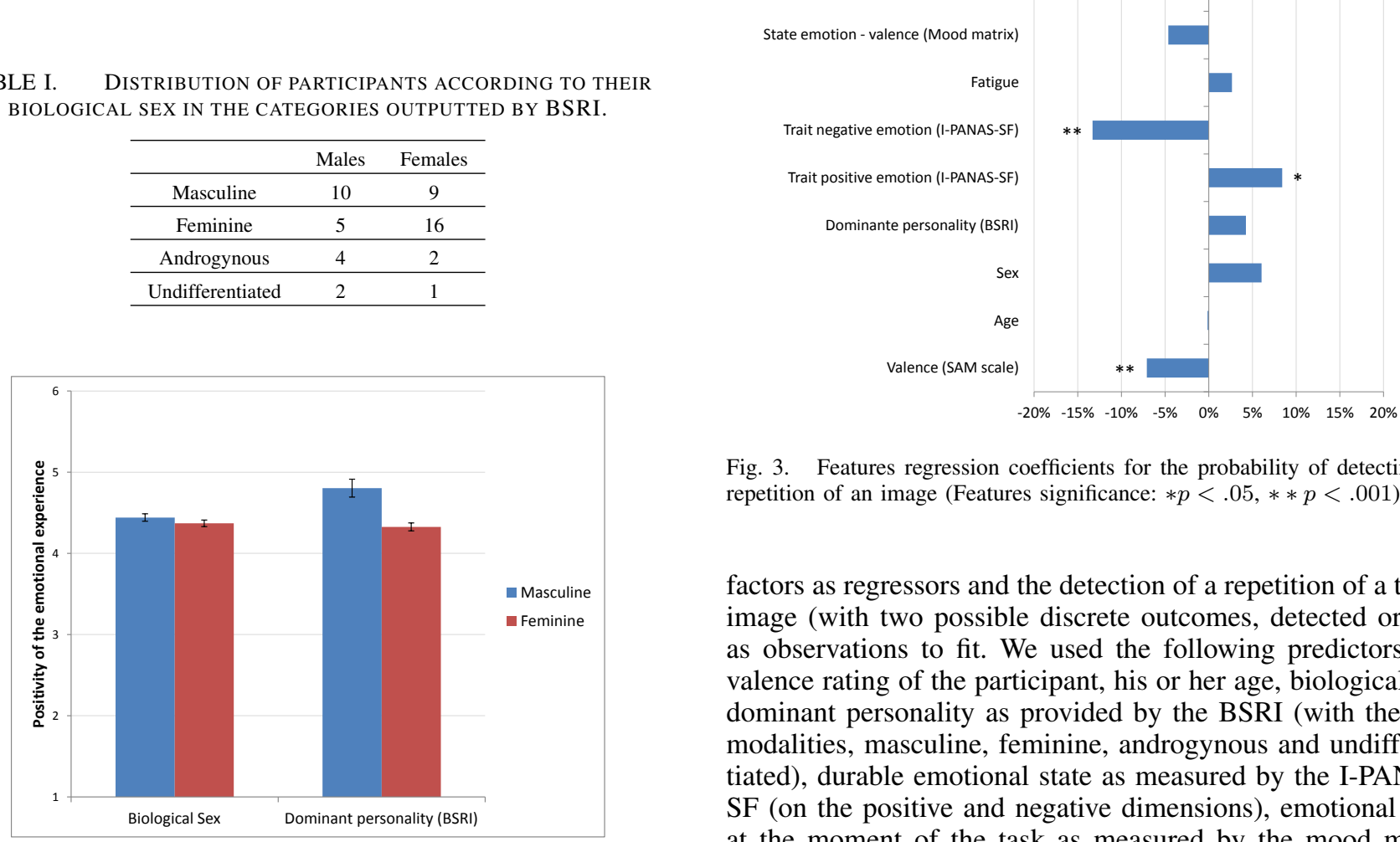

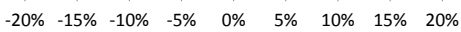

Fig. 3. Features regression coefficients for the probability of detecting the repetition of an image (Features significance: $* p<.05, * * p<.001$ ).

Fig. 2. Mean rating of the emotional experience in function of the biological sex and the dominant personality (error bars represent the standard errors of the mean).

\section{B. Biological sex and Dominant personality effects on emo- tional ratings}

We performed a two-way ANOVA for unbalanced design (because the number of observations at each factor-level combination were unequal) for testing the effects of biological sex and the dominant personality as assessed by the BSRI (with only the two categories Masculine and Feminine) on the ratings of positivity/negativity of the experiences (see Fig.2). We observed no significant effect of biological sex (male, female) on valence ratings $(F(1)=0.22, p=.641$ ). On the contrary, we observed a significant effect of the dominant personality (masculine, feminine) on valence ratings $(F(1)=5.29, p<.001)$, with individuals classified into 'Feminine' category who on average rated the pictures as more negative.

As the literature suggests that females could rate negative images as more negative than males do, because of their greater receptivity to negative stimuli [9], [10], we reproduced the same analysis only on the negative images (i.e. for which the mean valence score, calculated from the ratings of all participants, was under the value 5 , which corresponds scale to a neutral emotional state on the SAM scale). We observed a similar pattern, with no significant effect of biological sex on valence ratings of emotional experience induced by negative images $(F(1)=0.67, p=.412)$, but a significant effect of the dominant personality (masculine or feminine) $(F(1)=15.85, p<.001)$

\section{Regression analysis}

For the analysis of influential individual factors, we adopted a Logistic Regression, using the measured individual

factors as regressors and the detection of a repetition of a target image (with two possible discrete outcomes, detected or not) as observations to fit. We used the following predictors: the valence rating of the participant, his or her age, biological sex, dominant personality as provided by the BSRI (with the four modalities, masculine, feminine, androgynous and undifferentiated), durable emotional state as measured by the I-PANAS$\mathrm{SF}$ (on the positive and negative dimensions), emotional state at the moment of the task as measured by the mood matrix (on the arousal and valence dimensions), and level of fatigue.

The model, in case of a single observation, can be written as:

$$
y_{n}=\beta_{0}+\sum_{i=1}^{n} x_{n k} \beta_{k}+\epsilon_{n}
$$

where $y$ is the dependent variable - the hit or miss in the detection of the repetition of the image,$- x$ are our predictor values, $\beta$ are the coefficients to be estimated, and $\epsilon$ indicates the error term. Even if we expect our model to be more complex than linear, we adopted this method to have interpretable outputs for our features.

The results of the Logistic Regression fit are shown in Fig 3. Each computed coefficient expresses the influence of an individual factor on the relative chance that a repetition of an image is detected rather than missed. The method also provided a measure of the statistical significance of each feature in the model via their $p$-value.

In the resulting model, the more positive emotional experience induced by the image is, the less the repetition of the image is likely to be detected. About long-term emotional state, the more positive it is, the more the repetition of an image is likely to be detected, and the more negative it is, the less the repetition of an image is likely to be detected. Finally, the higher the arousal level reported by the participant about his mood at the moment of the task, the more the repetition of an image is likely to be detected. We found no statistically significant effect of age, biological sex, dominant personality, valence of mood at the moment of the task or level of fatigue on the chance for a repeated image to be recognized.

To test our mathematical model, we used a repeated random sub-sampling cross-validation (with $75 \%$ of data - i.e. 3875 observations - used for training, and $25 \%$ for classification, for each of the 40 splits). We obtained a classification accuracy of $66 \%$, with confidence interval of $3 \%$. 


\section{Support vector machine}

As underlined in [25], Logistic Regression might result into low accuracies, so it is interesting to complete it with a Support Vector Machine (SVM), which is able to model more complex relations between factors (with the shortcoming that mathematical functions linking inputs and outputs are not easily explainable). In this study, we used a SVM to obtain a baseline, in order to assess the relative efficiency of our model. We adopted a SVM with linear kernel as classifier, using the same data and cross-validation method as in Logistic Regression. We obtained a classification accuracy of $71 \%$, with confidence interval of $3 \%$. This performance gives an insight of how well our regression model fit the data. By losing a bit of performance using regression model rather than a SVM, we nevertheless gain the possibility to interpret the relations.

\section{DisCUSSION}

\section{A. Biological sex and Dominant personality effect on emo- tional ratings}

We found no significant difference between males and females in the way they rate positivity/negativity of the experience induced by an image. On the contrary, we found a significant difference between individuals with a masculine dominant personality and those with a feminine dominant personality (according to the BSRI), in the way individuals in Feminine category rated as more negative in general the emotional experiences induced by images. This suggests that personality has a greater impact than biological sex on the way an individual experiences an emotion.

We observed the same pattern (i.e. no effect of biological sex) for negative images, when literature suggests that females feel in general with more intensity the negative experiences (e.g. [9], [10]). This seems to be a lack of power problem, since we showed that males are more often classified into Masculine personality and females into Feminine category, and that the masculinity-femininity has an impact on the way valence of the experience is rated. The way we measured the valence of the experience induced by images - i.e. with self-report instrument - could explain a lack of power (the aforementioned studies used direct measures, namely ERPs and Skin Conductance Responses for Lithari et al., fIRM for Wrase et al.).

More generally, the interpretation of BSRI categorizations must be taken with caution: among others, the items are based on gender stereotypes in the American society of the seventies, whose relevance in French society today could be discussed.

\section{B. Modeling}

In our regression model, when valence of the experience increases, the probability to detect the repetition of an image decreases, suggesting that negative images are better memorized than others. It has long been known that positive and negative informations are better recognized than neutral ones, suggesting a nonlinear relation between valence and memorability of the images. However, some studies with young adults found that negative information is remembered better than positive information (e.g. [26]-[28]), which is consistent with our results. This negativity bias in memory could disappear with age, as suggested in [6], in which the relative number of negative images compared with positive and neutral images recalled decreased with age. About that, it would be interesting to work with older adults than those who passed our experiment (our oldest participant was 41).

When the participant's negative trait emotions increased, the probability to recognize the repeated image decreased; on the contrary, when the participant's positive trait emotions increased, this probability increased. This suggests that negative long-term mood negatively impacts the memory, while a positive mood is rather beneficial; this result is consistent with the fact that depression is often associated with poor memory in general (reviewed by [29]).

We found that when the arousal level of the emotional state at the moment of the task increased, the probability for a repeated image to be detected also increased, while we found no significant effect for the valence. These results suggest that when an individual is aroused, he has a better memory performance for the image recognition, while the positivity/negativity of its transitory emotional state do not impact, or impact less, this performance. About it, we can talk about potential confounding variables, in particular attention and information processing, associated both with arousal and memory [30], which maybe acted as intermediate variables.

Maybe because their relations with recognition memory are not linear, or because of a too limited number of participants in our study, we found no significant effect of age, biological sex, BSRI personality or fatigue on the probability to detect a repeated image using Logistic Regression. For age, the aforementioned fact that only young adults passed our experiment is a shortcoming; indeed, if memory performance decreases in normal aging [31], this phenomenon does not occur generally before a certain age. About biological sex and dominant personality, we could expect (from the literature and our ANOVA results, respectively) that these variables would play a role in memory for emotional images, because of the different ways these different categories of individuals could experience emotion, and the link between emotion and memory. This absence of significant result is maybe due to the fact that the effect is tenuous, or too complex to be effectively captured by the analysis method we adopted. About fatigue, it sounds from our results that a moderate fatigue (only three participants have reported a low level of fatigue - i.e. less than three on the fatigue scale) does not critically impact memory, such as a great fatigue might do.

\section{CONCLUSION}

Memorability can be considered as a relevant information in personalized multimedia optimization. In this paper, we focused on individual factors (in particular emotional) it is relevant to take into account to improve the memorability of an experience induced by a digital image. Real-time individual data acquisition is currently booming, in particular emotional data acquisition (under the impulse of recent research interests, such as Affective computing), promising to provide in the near future new efficient tools to capture individual differences in multimedia experience preferences. To take advantage of these technological advancements, it is important that new studies focus on understanding the impact of user factors on multimedia experience appreciation, and develop methods for 
integrating user information in multimedia system optimization.

\section{REFERENCES}

[1] P. Isola, J. Xiao, A. Torralba, and A. Oliva, "What makes an image memorable?" in 2011 IEEE Conference on Computer Vision and Pattern Recognition (CVPR), Jun. 2011, pp. 145-152.

[2] M. Mancas and O. L. Meur, "Memorability of natural scenes: The role of attention," in 2013 IEEE International Conference on Image Processing, Sep. 2013, pp. 196-200.

[3] P. Isola, J. Xiao, D. Parikh, A. Torralba, and A. Oliva, "What Makes a Photograph Memorable?" IEEE Transactions on Pattern Analysis and Machine Intelligence, vol. 36, no. 7, pp. 1469-1482, Jul. 2014.

[4] A. Khosla, J. Xiao, A. Torralba, and A. Oliva, "Memorability of image regions," in Advances in Neural Information Processing Systems, 2012, pp. $305-313$.

[5] D. Joshi, R. Datta, E. Fedorovskaya, Q.-T. Luong, J. Wang, J. Li, and J. Luo, "Aesthetics and Emotions in Images," IEEE Signal Processing Magazine, vol. 28, no. 5, pp. 94-115, Sep. 2011.

[6] S. T. Charles, M. Mather, and L. L. Carstensen, "Aging and emotional memory: The forgettable nature of negative images for older adults," Journal of Experimental Psychology: General, vol. 132, no. 2, pp. 310324, 2003.

[7] D. Grühn and S. Scheibe, "Age-related differences in valence and arousal ratings of pictures from the International Affective Picture System (IAPS): Do ratings become more extreme with age?" Behavior Research Methods, vol. 40, no. 2, pp. 512-521, May 2008.

[8] J. A. Mikels, G. R. Larkin, P. A. Reuter-Lorenz, and L. L. Carstensen, "Divergent trajectories in the aging mind: Changes in working memory for affective versus visual information with age," Psychology and Aging, vol. 20 , no. 4 , pp. $542-553,2005$

[9] J. Wrase, S. Klein, S. M. Gruesser, D. Hermann, H. Flor, K. Mann, D. F. Braus, and A. Heinz, "Gender differences in the processing of standardized emotional visual stimuli in humans: a functional magnetic resonance imaging study," Neuroscience Letters, vol. 348, no. 1, pp. 41-45, Sep. 2003.

[10] C. Lithari, C. A. Frantzidis, C. Papadelis, A. B. Vivas, M. A. Klados, C. Kourtidou-Papadeli, C. Pappas, A. A. Ioannides, and P. D. Bamidis, "Are Females More Responsive to Emotional Stimuli? A Neurophysiological Study Across Arousal and Valence Dimensions," Brain Topography, vol. 23, no. 1, pp. 27-40, Dec. 2009.

[11] J. S. Eccles, J. E. Jacobs, and R. D. Harold, "Gender Role Stereotypes, Expectancy Effects, and Parents' Socialization of Gender Differences,' Journal of Social Issues, vol. 46, no. 2, pp. 183-201, Jul. 1990.

[12] S. L. Bem, "On the utility of alternative procedures for assessing psychological androgyny," Journal of Consulting and Clinical Psychology, vol. 45, no. 2, pp. 196-205, 1977.

[13] S. Bem, Bem sex-role inventory: Professional manual. Consulting Psychologists Press, 1981.

[14] P. C. Watkins, A. Mathews, D. A. Williamson, and R. D. Fuller, "Moodcongruent memory in depression: Emotional priming or elaboration?' Journal of Abnormal Psychology, vol. 101, no. 3, pp. 581-586, 1992.

[15] S. Drace, "Evidence for the role of affect in mood congruent recall of autobiographic memories," Motivation and emotion, vol. 37, no. 3, pp. 623-628, 2013

[16] D. Hermans, J. D. Houwer, and P. Eelen, "The affective priming effect: Automatic activation of evaluative information in memory," Cognition and Emotion, vol. 8, no. 6, pp. 515-533, Nov. 1994.

[17] R. P. Hart, D. G. Buchsbaum, J. B. Wade, R. M. Hamer, and J. A. Kwentus, "Effect of sleep deprivation on first-year residents' response times, memory, and mood." Academic Medicine, vol. 62, no. 11, pp $940-2,1987$

[18] P. J. Lang, M. M. Bradley, and B. N. Cuthbert, "International affective picture system (IAPS): Affective ratings of pictures and instruction manual," Technical report A-8, 2008.

[19] E. R. Thompson, "Development and Validation of an Internationally Reliable Short-Form of the Positive and Negative Affect Schedule (PANAS)," Journal of Cross-Cultural Psychology, vol. 38, no. 2, pp. 227-242, Mar. 2007
[20] D. Watson, L. A. Clark, and A. Tellegen, "Development and validation of brief measures of positive and negative affect: The PANAS scales," Journal of Personality and Social Psychology, vol. 54, no. 6, pp. 10631070, 1988.

[21] E. Eich and J. Metcalfe, "Mood dependent memory for internal versus external events," Journal of Experimental Psychology: Learning, Memory, and Cognition, vol. 15, no. 3, pp. 443-455, 1989.

[22] W. Revelle and D. A. Loftus, "The implications of arousal effects for the study of affect and memory," The handbook of emotion and memory: Research and theory, pp. 113-149, 1992.

[23] M. M. Bradley and P. J. Lang, "Measuring emotion: The self-assessment manikin and the semantic differential," Journal of Behavior Therapy and Experimental Psychiatry, vol. 25, no. 1, pp. 49-59, Mar. 1994.

[24] J. T. Spence, R. Helmreich, and J. Stapp, "Ratings of self and peers on sex role attributes and their relation to self-esteem and conceptions of masculinity and femininity," Journal of Personality and Social Psychology, vol. 32, no. 1, pp. 29-39, 1975.

[25] G. Mohammadi and A. Vinciarelli, "Automatic Personality Perception: Prediction of Trait Attribution Based on Prosodic Features," IEEE Transactions on Affective Computing, vol. 3, no. 3, pp. 273-284, Jul. 2012

[26] S. A. Dewhurst and L. A. Parry, "Emotionality, distinctiveness, and recollective experience," European Journal of Cognitive Psychology, vol. 12 , no. 4 , pp. 541-551, Dec. 2000.

[27] M. Mather, E. Shafir, and M. K. Johnson, "Misremembrance of Options Past: Source Monitoring and Choice," Psychological Science, vol. 11, no. 2, pp. 132-138, Mar. 2000.

[28] K. N. Ochsner, "Are affective events richly recollected or simply familiar? The experience and process of recognizing feelings past," Journal of Experimental Psychology: General, vol. 129, no. 2, pp. 242261, 2000 .

[29] D. B. Burt, M. J. Zembar, and G. Niederehe, "Depression and memory impairment: A meta-analysis of the association, its pattern, and specificity," Psychological Bulletin, vol. 117, no. 2, pp. 285-305, 1995.

[30] S.-A. Christianson, The Handbook of Emotion and Memory: Research and Theory. Psychology Press, Feb. 2014.

[31] T. Hedden and J. D. E. Gabrieli, "Insights into the ageing mind: a view from cognitive neuroscience," Nature Reviews Neuroscience, vol. 5, no. 2, pp. 87-96, Feb. 2004. 